\title{
3D VR Serious Games for Production \& Logistics
}

\author{
A. Boden ${ }^{1}$, A. Buchholz ${ }^{1}$, M. Petrovic ${ }^{1}$, F.J. Weiper ${ }^{1}$
}

\begin{abstract}
Within the Institute of Production of the University of Applied Science Cologne, TH Köln, the logistics IT group has initiated a new project for the 3D Virtual Reality digitization of logistics and manufacturing processes. The 3D Virtual Reality Serious Games learning environment is in accordance with the real physical model factory at the institute, where students of business engineering classes study and exercise the interdisciplinary processes of a whole manufacturing unit. On the basis of this project initiation within the institute of production we want to build and offer a widespread open-source standard framework for programming 3D VR Serious Games for Production \& Logistics that can be used by other universities and industrial partners. A sneak preview of the game can be viewed in [1].
\end{abstract}

\section{Keywords}

Serious Game, 3D Virtual Reality, Production, Logistics

\section{Introduction}

The topic of 3D Virtual Reality in Industrial context has raised lot of interest in the past few years. One of the most promising application areas is the concept of 3D Serious Games in which on-the-job-trainings and advanced education in industrial context is performed with the help of 3D Virtual Reality games. By the immersive environment in which the trainee is placed to perform his training levels, not only the training performance is boosted by factors [2]. If we compare the results against traditional trainings, the virtual reality concept also can save time and resources to a large extend. Trainings can be performed without expensive real hardware training environments, machines, or production processes. The on-the-job-trainings can be performed without disturbance of the real production. Nonetheless trainings can easily be repeated, can be transferred to distributed training centers across the world. Finally, the training environments can easily be adapted to new scenarios and learning outcomes, since we do not need to change and arrange physical hardware but a software program only. The landscape for Serious Games in industry worldwide is growing rapidly, and because of the rationalism, the industry in any segment is adapting in very fast manner. For the entire 3D Virtual Reality scene, the market growth is measured in magnitudes of billions of Euros for the upcoming decades [3].

1 Institute for Production, University of Applied Science Cologne, TH Köln 


\section{3D Serious Games for Production \& Logistics}

For university learning platforms serious games can also be very attractive. The logistics IT group of the University of Applied Science Cologne, TH Köln, has initiated a project for the 3D Virtual Reality digitization of logistics and manufacturing processes. The 3D Virtual Reality learning environment is in accordance with the real physical model factory, where students of business engineering classes exercise the interdisciplinary processes of a whole manufacturing unit [4].

Within the model factory there is installed on firsthand a fully equipped IT process that manages the entire flow from the customer request down to the production confirmation and product quality checks. The hardware equipment includes an automated storage device, a production line, CNC controlled milling machine, a laser-based gravure printer, a cutting machine, robotic arms for picking and material movement, a quality measurement work bench, a view of the plant can be seen in fig. 1 .

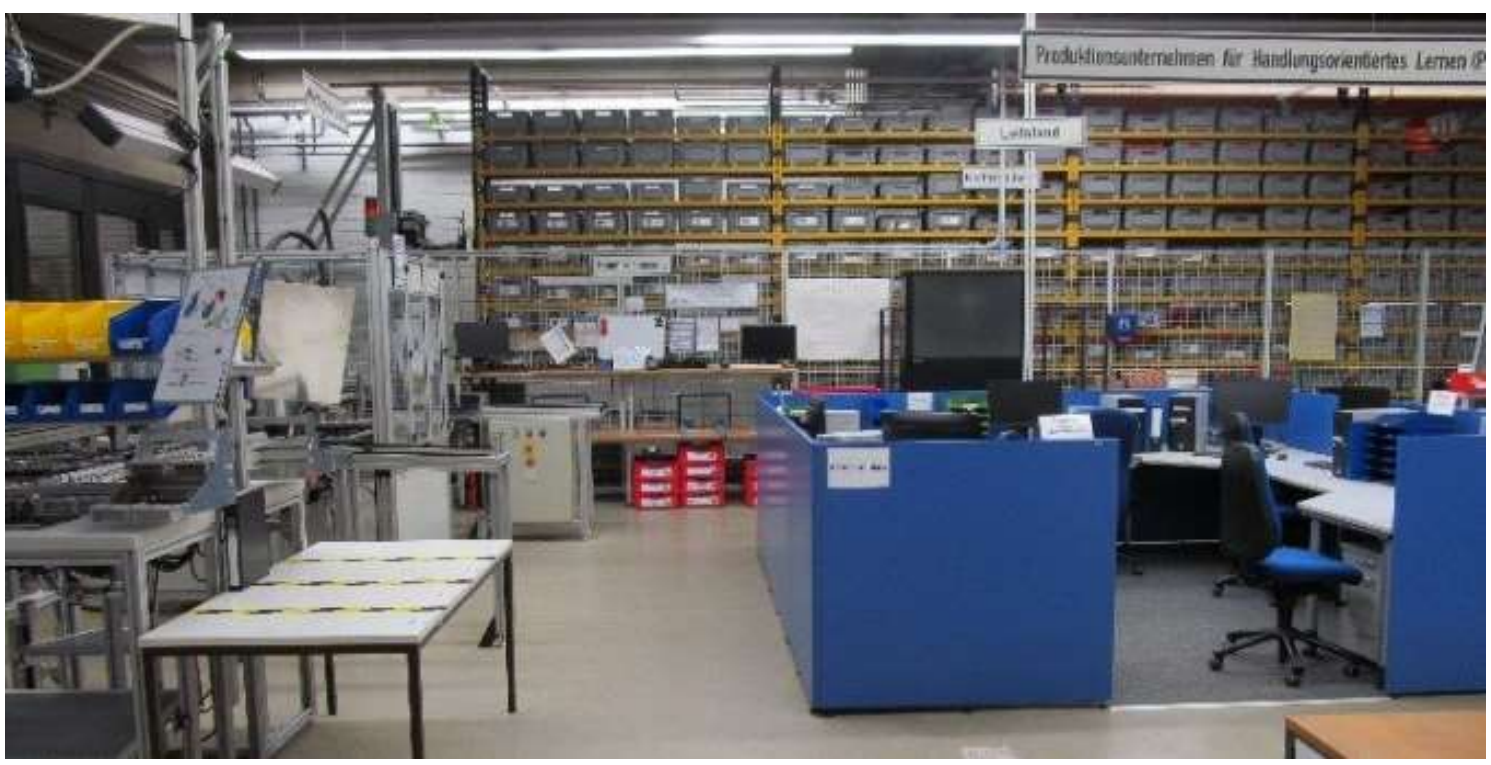

Figure 1. Layout of the model plant at the Institute for Production at UAS Cologne, TH Köln. Further details can be found in [3].

The machinery is of high quality and very expensive industrial hardware. This means, that the students will need a lot of introductions, safety instruction, training, and exercises, before they can start the actual challenges of their lessons and courses. The new project now depicts in 3D Virtual Reality the entire hall with the equipment, and students have an excellent environment to study the entire manufacturing processes and machinery, before starting the hands-on exercises in the real physical world.

The 3D VR Serious Games are programmed on the basis of open-source standard frameworks. For the development of the Games we use the framework Unity [5] with the runtime environment Steam VR, components for the usage within the 3D Virtual Reality Game are being modelled with the 3D Graphics studio Blender [6]. For the 3D VR visualization high performance 3D VR glasses from HTC Vive Pro are being introduced. 


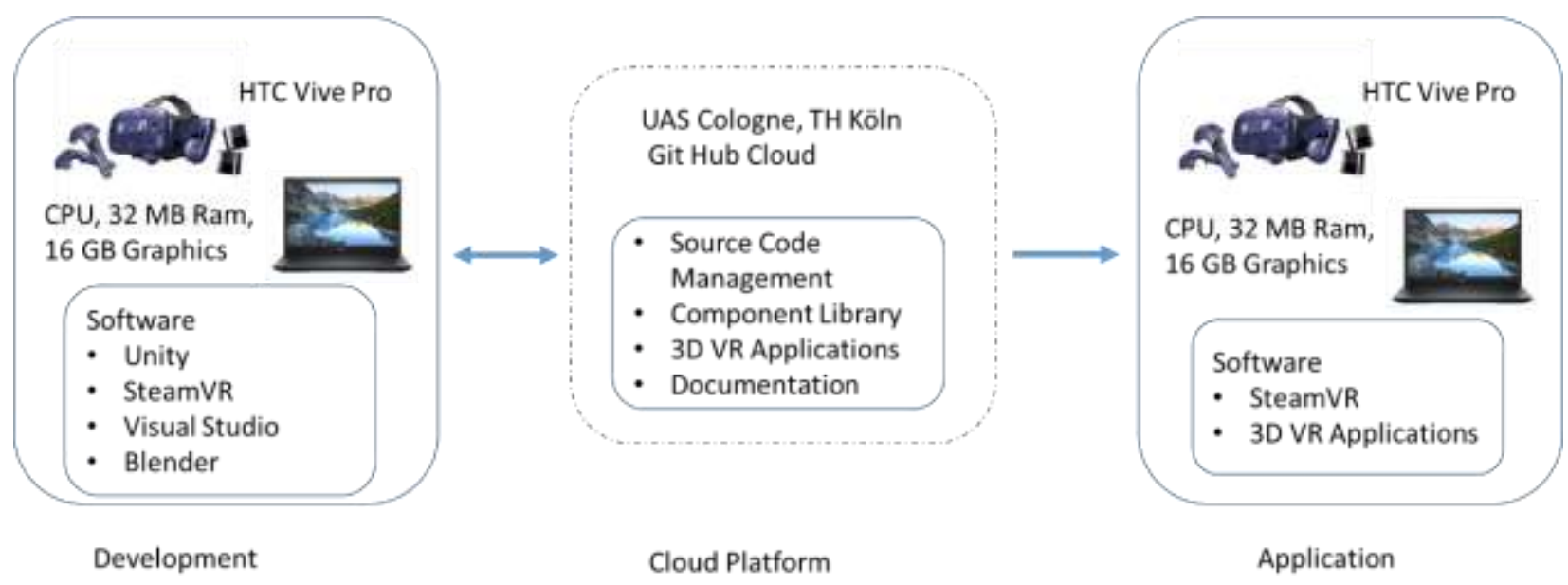

Figure 2. The Architecture for 3D VR Serious Games for Production \& Logistics

In fig. 2 the chosen architecture for the 3D VR environment is depicted. The game includes the full production \& logistics process, i.e. the IT workflow, safety instructions, instructions for the machinery \& manual assembly steps, as well as the instruction for the automated storage device. Further within the 3D VR Serious Game audio streams and video clips are embedded for the sake of instructions and documentation. In the first phase of the project there is a scenario depicted, where different types of pumps (e.g. peristaltic \& centrifugal pumps), are being manufactured. The workflow starts with order creation and confirmation. The next step is the material supply for the production out of the automated storage device, followed by the assembly of the pumps at a separate assembly work bench. Finally, the pumps are being tested on a quality measurement workbench. Fig. 3 depicts sample views from the 3D VR Serious Game.

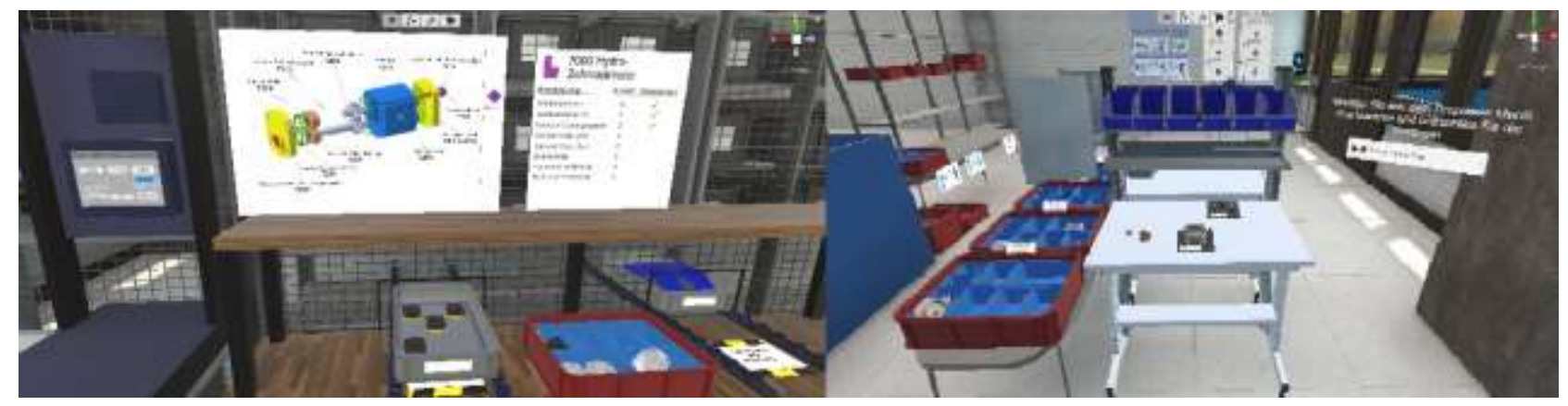

Figure 3. The picture shows details of the automated storage device (left), and the assembly workbench (right)

\section{Outlook}

On the basis of this project initiation within the institute of production we want to build and offer a widespread open-source standard framework for programming 3D VR Serious Games for Production \& Logistics that can be used by other universities and industrial partners. We already have developed for the current programs at hand an extended library of components to be used, like machines, production lines, desktops, cases, shelfs, storage device, pumps, screws \& nuts, tools, meters, etc... to depict realistic manufacturing scenes. With the help of CAD software and procedures in 3D graphics suites like Blender, we will integrate more and more components for the usage within new scenarios. On top 
of that we have a library of patterns, for example to steer the workflows, to interact with hand controllers, or to include audio streams and videos for instructions. Finally, there is a refined organization of the whole development environment that enables new developers to easily step into the framework and start developing new scenarios for other processes in Production \& Logistics context. In a next step we will include the possibility of online interfaces with the real-world equipment to capture data also from the physical processes. By that, we will enter the research area of remotely controlled automated processes. This new field will need additional routines, new organizational structures within the framework, and a high-speed industrial framework for standardized interfaces.

\section{Literature}

[1] A. Boden, F.J. Weiper, TH Köln VR Modellfabrik, https://youtu.be/N7ZzIA31co8, 2021

[2] S. Kaczmarek, M. Henke, Gamification in der Logistik. Huss-Verlag GmbH, 2017; R. Dörner, S. Göbel, W. Effelsberg, J. Wiemeyer, (eds.). Serious Games: Foundations, Concepts and Practice. Springer 2016

[3] PWC, German Entertainment \& Media Outlook 2019 - 2023, Virtual Reality in Deutschland, 2019

[4] Model Factory at the UAS Cologne, TH Köln, https://www.th-koeln.de/en/automotive-systems-and-production/modellfabrik-model-factory 72685.php, 2020

[5] T. Theiss, Einstieg in Unity, Rheinwerk Verlag, 2021

[6] O. Baechler, X. Greer, Blender 3D by Example, Packt Publishing 2020 